\title{
Application of NASA General-Purpose Solver to Large-Scale Computations in Aeroacoustics
}

\author{
Willie R. Watson* and Olaf O. Storaasli ${ }^{\dagger}$ \\ NASA Langley Research Center, Hampton, VA 23681, USA
}

\begin{abstract}
Of several iterative and direct equation solvers evaluated previously for computations in aeroacoustics, the most promising was the NASA-developed General-Purpose Solver (winner of NASA's 1999 software of the year award). This paper presents detailed, single-processor statistics of the performance of this solver, which has been tailored and optimized for large-scale aeroacoustic computations. The statistics, compiled using an SGI ORIGIN 2000 computer with 12 Gb available memory (RAM) and eight available processors, are the central processing unit time, RAM requirements, and solution error. The equation solver is capable of solving 10 thousand complex unknowns in as little as $0.01 \mathrm{sec}$ using $0.02 \mathrm{~Gb}$ RAM, and 8.4 million complex unknowns in slightly less than 3 hours using all $12 \mathrm{~Gb}$. This latter solution is the largest aeroacoustics problem solved to date with this technique. The study was unable to detect any noticeable error in the solution, since noise levels predicted from these solution vectors are in excellent agreement with the noise levels computed from the exact solution. The equation solver provides a means for obtaining numerical solutions to aeroacoustics problems in three dimensions.
\end{abstract}

\footnotetext{
* Senior Research Scientist, Aerodynamics, Aerothermodynamics, and Acoustics Competency; Computational Modeling and Simulation Branch; Mail Stop 128; Tel: (757)-864-5290; E-mail: W.R.Watson@LaRC.NASA.Gov; fax: (757)-864-8816

${ }^{\dagger}$ Senior Research Scientist, Stuctural and Materials Competency; Analytical and Computational Methods Branch; Mail Stop 474; Tel: (757)-864-2927; E-mail: O.O.Storaasl@LaRC.NASA.Gov; fax: (757)-864-8912
} 
keywords: finite element, Vector-Sparse Solver, General-Purpose Solver, impedance eduction, symmetric matrices, solver statistics, large-scale computations, aeroacoustics, SGI ORIGIN 2000.

\section{Introduction}

Fan noise is defined as unwanted acoustic energy that is generated at the fan face or guide vanes of a turbofan engine. Fan noise accounts for a significant portion of community noise radiated from conventional and high bypass ratio engines. Noise reduction research today focuses on reducing fan noise levels radiated from future aircraft by a factor of 2 relative to 1990 levels. Installation of acoustic treatment (i.e., liners) into the nacelles of aircraft engines remains one of the most effective means for achieving these noise reduction goals [1]. To this end, an accurate knowledge of liner impedance is critical in optimizing the treatment for maximum noise suppression. To date, much of the design effort has concentrated on expensive and time consuming experimental testing. In addition, experimental tests have not account for variable surface impedance that results naturally from imperfections in the liner manufacturing process. An accurate numerical model could predict the lining impedance in a less costly and more time-efficient manner, and at the same time account for surface impedance variability.

In a recent paper [2] a numerical method for extracting the impedance of an acoustic material was developed and validated for plane wave sources. In this approach, the timedependent acoustic equations are Fourier transformed into a single differential equation in frequency space. Source and exit boundary conditions for the numerical model are obtained from measurements in a flow duct that provides grazing-flow and grazing-incidence sound over the test liner. The frequency domain differential equation is then coupled to the measured boundary conditions and the solution is approximated by a standard finite element method. The finite element method leads to a large, sparse, indefinite linear system of complex equations. The acoustic impedance of the test liner is then educed by a series of 
successive corrections to an original estimate for the unknown impedance, the process being carried out repetitively until the corrected impedance reproduces the measured upper wall pressure. Each successive correction to the impedance is determined via an optimizer that uses the solution to the system of complex equations to construct the penalty function [2]. As this method has progressed to include higher frequencies, nonplanar sound sources and flow [3], the absence of an efficient (in time and memory) equation solver for this large system of complex acoustic equations has emerged as a major impediment to further development of the method. A time-and memory-efficient solver for aeroacoustics would allow numerical methods to be extended to high frequency sources and three-dimensional aeroacoustics computations.

Large systems of complex equations may be solved by iterative [4] or direct [5] methods. Recently, the performance of several iterative and direct equation solvers were evaluated to establish their suitability for computations in aeroacoustics [6]. Based on that study, the commercial version of the NASA-developed General-Purpose Solver (GPS) [7] emerged as the most promising solver. However, the conclusion derived in [6] was based solely on a study of the central processing unit time required by the solver. Among the other metrics that need to be considered in evaluation of the solver are memory requirements (RAM) and solution error. Additionally, for realistic aeroacoustics computations, the impedance spectrum of a test sample would be required for frequencies up to 25,000 $\mathrm{Hz}$ and 0.5 Mach number. A "back of the envelope calculation" at this frequency and Mach number shows that approximately 8 million equations need to be solved to resolve all propagating modes contained within the computational volume. Unfortunately, due to memory constraints the results presented in [6] were only small-scale calculations (i.e., less than 80,000 equations), and no attempt was made to obtain optimal statistics.

The purpose of this paper is to present detailed statistics of the performance of a vastly improved version of the GPS solver [8] that has been specifically tailored and optimized for nacelle aeroacoustics computations. The three metrics used in this evaluation are CPU time, 
RAM requirements, and solution error. These statistics are evaluated for unknowns ranging from 10 thousand to 8.4 million.

\section{Physical Problem Description}

Figure 1 shows a schematic of the two-dimensional duct used in this study. It should be noted, as suggested by Figure 1, that the math model discussed here is limited to a two-dimensional description that approximates a three-dimensional flow impedance tube as discussed in [3]. The axial and transverse directions are denoted by $x$ and $y$, respectively. The duct is $L$ units

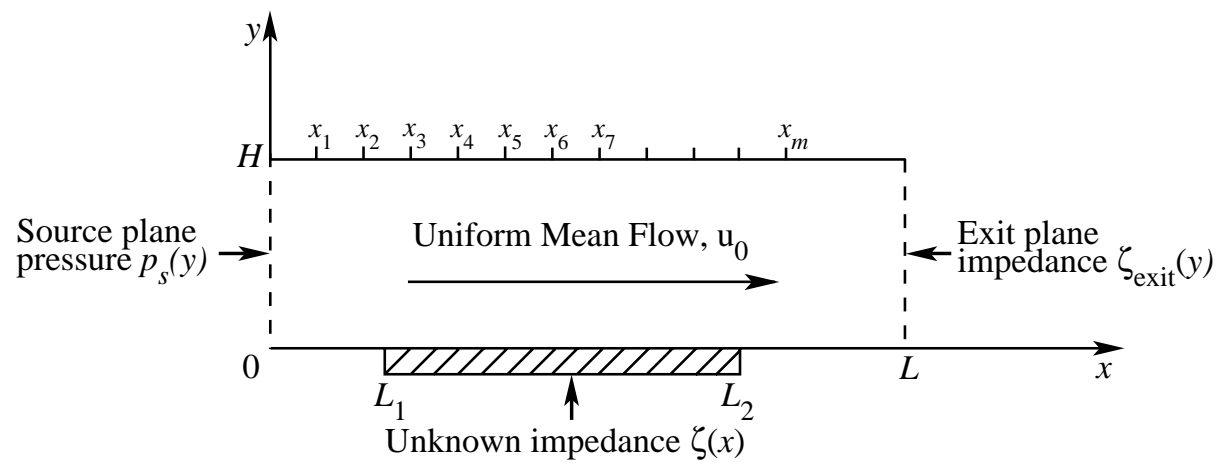

Fig. 1. Two-dimensional duct and coordinate system.

long, with the source and exit planes located at $x=0$ and $x=L$, respectively. Required inputs at the source and exit planes are the source pressure $p_{s}(y)$ and the normalized exit impedance $\zeta_{\text {exit }}(y)$, respectively. Throughout this work all impedances are normalized with respect to the characteristic impedance, $\rho_{0} c_{0}$, of the air in the duct, and the upper wall is rigid. Here $\rho_{0}$ and $c_{0}$ are the mean density and sound speed of air respectively, in the duct. Note that there is a mean flow in the axial direction that flows subsonically from left to right with uniform speed, $u_{0}$. Further, there are $m$ points, located at $x=x_{1}, x_{2}, x_{3}, \ldots, x_{m}$ along the upper wall, at which the acoustic pressures, $p\left(x_{I}, H\right)$ are assumed known. It should be noted that flow impedance tube apparatuses such as that discussed in [3] are routinely used to obtain measurement of the source pressure $p_{s}(y)$, exit impedance $\zeta_{\text {exit }}(y)$, and the upper wall acoustic pressures, $p\left(x_{I}, H\right)$, for impedance eductions.

The sound-absorbing material constitutes the part of the bottom wall of the duct between 
$L_{1} \leq x \leq L_{2}$. Outside this region, the lower wall is rigid. The acoustic material is assumed to be locally reacting (i.e., acoustic waves propagate through it only normal to the surface). The sound-absorbing material has an unknown normalized impedance $\zeta(x)$, as shown. The physical problem is to determine, from the measured boundary data, the impedance of the acoustic material as a function of the mean flow speed $u_{0}$.

\section{Governing Equations and Boundary Conditions}

The equations that describe the propagation of acoustic pressure disturbances through a duct containing a flowing fluid as depicted in Figure 1 are derived from the Navier-Stokes and energy equation, neglecting viscous and heat-conducting effects. The justification for the neglect of viscosity and heat conduction is that the passage of sound waves through a moving fluid is an isentropic process. Note that the equations that are the subject of this investigation result from the additional assumptions that

1. nonlinear acoustic effects can be neglected

2. the acoustic disturbance has reached a steady-state

3. the steady-flow is in uniform motion

Thus, the mathematical problem is to find the solution to the convected wave equation [9]

$$
\begin{gathered}
\left(1-M_{0}^{2}\right) \frac{\partial^{2} p}{\partial x^{2}}+\frac{\partial^{2} p}{\partial y^{2}}-2 i k M_{0} \frac{\partial p}{\partial x}+k^{2} p=0 \\
i=\sqrt{-1} ; \quad k=\frac{2 \pi f}{c_{0}} ; \quad M_{0}=\frac{u_{0}}{c_{0}}
\end{gathered}
$$

where $p(x, y)$ is the complex acoustic pressure in the duct and $f$ is the sound source frequency in hertz. The source, exit, and upper wall boundary conditions are [3]

$$
\begin{gathered}
p(0, y)=p_{s}(y) \\
\frac{\partial p(L, y)}{\partial x}=-\frac{i k p(L, y)}{\left[M_{0}+\zeta_{\text {exit }}(y)\right]}
\end{gathered}
$$




$$
\frac{\partial p(x, H)}{\partial y}=0
$$

while the correct form of the wall impedance boundary condition in the presence of the flowing fluid has been derived in $[10]$

$$
\frac{\partial p(x, 0)}{\partial y}=i k \frac{p(x, 0)}{\zeta(x)}+2 M_{0} \frac{\partial}{\partial x}\left[\frac{p(x, 0)}{\zeta(x)}\right]+\frac{M_{0}^{2}}{i k} \frac{\partial^{2}}{\partial x^{2}}\left[\frac{p(x, 0)}{\zeta(x)}\right]
$$

Together, Equation (1) and the boundary conditions, Equations (3)-(6), form a boundary value problem that can be solved to determine uniquely the upper wall pressures $p\left(x_{I}, H\right)$ for a given impedance function. Conversely, if the upper wall pressures $p\left(x_{I}, H\right)$, are known (i.e., measured), there is a unique wall impedance function $\zeta(x)$ that will reproduce these wall pressures. This impedance function is the unknown impedance of the test liner.

Unfortunately, exact solutions for the wall impedance function satisfying the boundary value problem do not exist for an arbitrary set of boundary data, $p_{s}(y)$, $\zeta_{\text {exit }}(y)$, and $p(x, H)$. Thus, the goal of impedance eduction techniques is to devise a numerical procedure for determining this unknown liner impedance in the presence of the flowing fluid. In a recently developed method $[2,3]$, the unknown acoustic impedance function $\zeta(x)$ of the test liner is "educed" by a series of successive corrections to an original estimate for the unknown impedance, the process being carried out repetitively until the corrected impedance reproduces the known upper wall pressure. Each successive correction to the impedance is determined by repeated numerical solutions to the nacelle acoustics boundary value problem defined by Equation (1) and boundary condition Equations (3)-(6).

The process of obtaining the numerical solution to the boundary value problem consists of two stages. In the first stage the continuous partial differential equation and boundary conditions are converted into a system of discrete algebraic equations. The second stage requires an equation solver to obtain the solution to the system of discrete algebraic equations. A number of methods are available for converting the continuous differential equations and boundary conditions into a system of discrete algebraic equations. The method used here is the finite element method. 


\section{Formulation of Discrete Equation System}

The continuous partial differential equation and boundary conditions are converted into a system of discrete algebraic equations using the finite element method. As shown in Figure 2, $N$ and $M$ points are used in the $x$ and $y$ directions, respectively. Note that the computational domain has been decomposed into a total of $(N-1) x(M-1)$ rectangular elements, as shown in the figure. Linear and cubic Hermite polynomial basis functions are

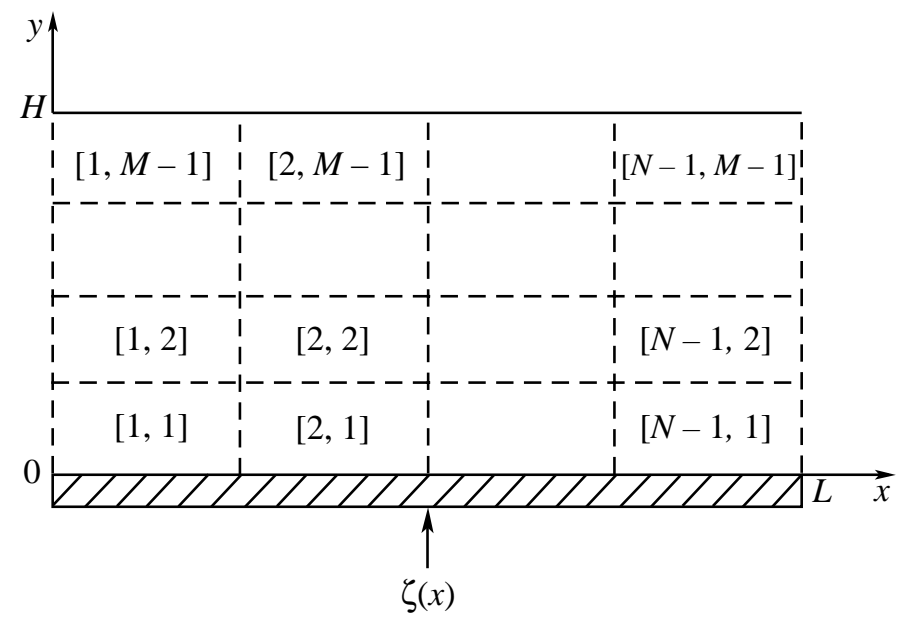

Fig. 2. Finite element discretization of two-dimensional duct.

used with and without flow, respectively. Implementations of the finite element method are given in detail elsewhere $[2,3]$ and are not discussed further in this paper. The finite element method leads to a discrete set of complex linear equations of the form

$$
[A(\zeta(x))]\{\Phi\}=\{F\}
$$

When the duct does not contain flow (i.e., $M_{0}=0$ ), the coefficient matrix is of the form

$$
[A(\zeta(x))]=\left[\begin{array}{rrrrr}
{\left[A_{1}\right]} & {\left[B_{2}\right]} & & & \\
{\left[B_{2}\right]^{T}} & {\left[A_{2}\right]} & {\left[B_{3}\right]} & & \\
& \ddots & \ddots & \ddots & \\
& & {\left[B_{N-1}\right]^{T}} & {\left[A_{N-1}\right]} & {\left[B_{N}\right]} \\
& & & {\left[B_{N}\right]^{T}} & {\left[A_{N}\right]}
\end{array}\right]
$$


where the superscript $T$ denotes matrix transpose and

$$
\begin{gathered}
{\left[B_{I}\right]=\left[\begin{array}{rrrrr}
a_{1}^{I} & b_{1}^{I} & & & \\
c_{1}^{I} & a_{2}^{I} & b_{2}^{I} & & \\
& \ddots & \ddots & \ddots & \\
& & c_{M-1}^{I} & a_{M-1}^{I} & b_{M-1}^{I} \\
& & & c_{M}^{I} & a_{M}^{I}
\end{array}\right]} \\
{\left[A_{I}\right]=\left[\begin{array}{rrrrr}
d_{1}^{I} & e_{1}^{I} & & & \\
e_{1}^{I} & d_{2}^{I} & e_{2}^{I} & & \\
& \ddots & \ddots & \ddots & \\
& & e_{M-1}^{I} & d_{M-1}^{I} & e_{M-1}^{I} \\
& & & e_{M-1}^{I} & d_{M}^{I}
\end{array}\right]}
\end{gathered}
$$

where $a_{J}^{I}, b_{J}^{I}, c_{J}^{I}, d_{J}^{I}$, and $e_{J}^{I}$ are complex coefficients. Thus, the zero flow matrix $[A(\zeta(x))]$, has the following properties

1. It is an $(N M) x(N M)$ indefinite matrix (i.e., construction of $[L]$ and $[U]$ requires row or column interchanges in $[A]$ )

2. It is complex and symmetric

3. Each major block $\left(\left[A_{I}\right],\left[B_{I}\right]\right)$ is an $M x M$ complex tridiagonal matrix and the diagonal blocks $\left(\left[A_{I}\right]\right)$ are symmetric

4. It is banded with a bandwidth of $(2 M+3)$

5. It is very sparse (i.e., only four of the $(M+1)$ superdiagonals contain nonzero elements) When the duct does contain flow (i.e., $M_{0} \neq 0$ ), the coefficient matrix is of the form

$$
\begin{gathered}
{[A(\zeta(x))]=\left[\begin{array}{rrrrr}
{\left[A_{1}\right]} & {\left[B_{2}\right]} & & & \\
{\left[C_{2}\right]} & {\left[A_{2}\right]} & {\left[B_{3}\right]} & & \\
& \ddots & \ddots & \ddots & \\
& & {\left[C_{N-1}\right]} & {\left[A_{N-1}\right]} & {\left[B_{N}\right]} \\
& & & {\left[C_{N}\right]} & {\left[A_{N}\right]}
\end{array}\right]} \\
{\left[B_{I}\right]=\left[\begin{array}{rrrrr}
{\left[a_{1}^{I}\right]} & {\left[b_{1}^{I}\right]} & & & \\
{\left[c_{1}^{I}\right]} & {\left[a_{2}^{I}\right]} & {\left[b_{2}^{I}\right]} & & \\
& \ddots & \ddots & \ddots & \\
& & {\left[c_{M-2}^{I}\right]} & {\left[a_{M-1}^{I}\right]} & {\left[b_{M-1}^{I}\right]} \\
& & & {\left[c_{M-1}^{I}\right]} & {\left[a_{M}^{I}\right]}
\end{array}\right]}
\end{gathered}
$$


where $\left[a_{J}^{I}\right],\left[b_{J}^{I}\right]$, and $\left[c_{J}^{I}\right]$ are $4 x 4$ complex matrices. When the nacelle contains flow, the structure of $\left[A_{I}\right]$ and $\left[C_{I}\right]$ are identical to that of $\left[B_{I}\right]$, but the complex coefficients in $\left[A_{I}\right]$ and $\left[C_{I}\right]$ are distinct from those of $\left[B_{I}\right]$. Thus, the flow matrix $[A(\zeta(x))]$, has the following properties

1. It is an $(4 N M) x(4 N M)$ indefinite matrix

2. It is complex and nonsymmetric

3. Each major block is a $4 M x 4 M$ complex matrix that is block tridiagonal

4. It is banded with a bandwidth of $8(M+2)-1$

5. It is very sparse

Much practical importance arises from the structure of $[A(\zeta(x))]$, as it is convenient for minimizing storage and maximizing computational efficiency. Approximately $98 \%$ of the computer resources required to educe the impedance are consumed in finding the solution to the discrete system. Considerable savings in computer resources are possible if the most

efficient solver is used. In [2] and [3], the solution to the discrete system is obtained by using a band solver. However, the band solver severely taxes RAM and CPU time by requiring storage and arithmetic operations on the inner null bands of $[A(\zeta(x))]$. This paper focuses on the use of an efficient sparse solver to obtain the solution of the system of discrete equations.

\section{The Equation Solver}

In this research effort, VSS [7] (a commercial version of NASA's GPS [8]) is exercised to obtain the solution to the aeroacoustic system defined by Equation (7). The NASA-developed GPS had its genesis in the need for large aerospace structures solutions in computational mechanics. GPS was subsequently extended to support matrices that are sparse or dense, positive definite or indefinite, real or complex. In addition, GPS has been extended to solve 
nonsymmetric matrices that are often generated, for example, in aeroacoustics problems containing flow.

The method of solution used by GPS and VSS is the commonly-used $[L][U]$ decomposition. A fairly general scheme exists for computing the lower triangular factorization matrix $[L]$ and the upper triangular factorization matrix $[U]$. One key innovation of the GPS soft-

ware is that $[L]$ and $[U]$ are computed cleverly, with due attention given to eliminating computations with zero elements while minimizing storage and CPU time. A second GPS innovation is a novel reordering method that retains the benefit of a multiple-minimum degree (MMD) reordering at a fraction of the MMD reordering time. This time reduction is accomplished by reordering a subset of the equations. The solver requires that only the nonzero coefficients in $[A(\zeta(x))]$ be stored in memory. The nonzero coefficients are stored in row format and as a single vector to facilitate the solution procedure. The GPS (and VSS) equation solver exploit the matrix characteristics (real or complex, symmetric or nonsymmetric, in-core or out-of-core) of the application and is designed to exploit the hardware features of current and future computers. Only a small fraction of the capability of the solver is used in this research effort (i.e., only the complex, symmetric, in-core capability was required). The equation solver used in this paper includes several recent innovations that are discussed in detail elsewhere [8]. The statistics computed in the following section are optimal in the sense that several of the solver software parameters, such as the "loop level" [7], have been optimized through numerical experimentation.

\section{Results}

An in-house computer code that constructs the coefficient matrix $[A(\zeta(x))]$ in the required NASA solver format has been linked to a commercial version of the GPS solver (VSS) in order to provide statistics for the aeroacoustics computations presented in this paper. The statistics for the solution of Equation (7) have been computed for both rigid-wall and soft-wall ducts, but in the absence of flow. Results were computed on an in-house SGI ORIGIN 2000 
computer that contained $12 \mathrm{~Gb}$ of available RAM and eight processors. All computations were performed on a single processor using double-precision (i.e., 64 bit) arithmetic with $M=N$.

Results are presented for a square duct $1 \mathrm{~m}$ in width $(L=H=1 \mathrm{~m})$ with the leading and trailing edges of the liner located at the source and exit planes, respectively $\left(L_{1}=0, L_{2}=L\right)$. Because the results computed here do not contain flow effects (i.e., $M_{0}=0$ ), only the symmetric version of the solver is used. All calculations presented in this paper are performed at standard atmospheric conditions. The source is a plane wave source $\left(p_{s}(y)=1\right)$ that oscillates at a frequency of $1000 \mathrm{~Hz}$ and the exit impedance is set to unity $\left(\zeta_{\text {exit }}=1\right)$. Because results in this paper have been purposely restricted to this range of parameters, exact solutions are available to check the solver error for the rigid-wall duct.

Figure 3 shows a plot of the CPU time and RAM required to solve for up to 8.4 million complex unknowns in a rigid-wall duct. Note that these statistics are plotted on a dual axis system with the CPU time referenced to the $y 1$ axis and the memory referenced to the $y 2$ axis. CPU times shown in the figure correspond to those required to obtain the solution vector (i.e., to forward and backward solve) after the coefficient matrix is constructed. Generally, the CPU time required for construction of the coefficient matrix or to obtain the "backward solve" is less than $2 \%$ of that required to obtain the solution vector. CPU times range from $0.01 \mathrm{sec}$ for 10 thousand complex unknowns to a maximum of nearly 3 hours for 8.4 million complex unknowns. The RAM ranges from $0.02 \mathrm{~Gb}$ for 10 thousand complex unknown to 12 Gb for 8.4 million. Note that the RAM scales linearly with the number of unknowns (RAM $\propto M N)$, whereas the CPU times scales with the $4 / 3$ power of the number of unknowns (CPU time $\left.\propto(M N)^{\frac{4}{3}}\right)$. It should be noted that the 8.4 million complex solution set consumed all of the RAM on the SGI ORIGIN 2000 and represents the largest number of complex unknowns that could be solved with this solver within the available RAM.

In order to check the accuracy of the solver solutions, the authors use the reduction in the noise level from the entrance to the exit of the duct (i.e., $\Delta \mathrm{dB}$ ) as a metric. This is a 


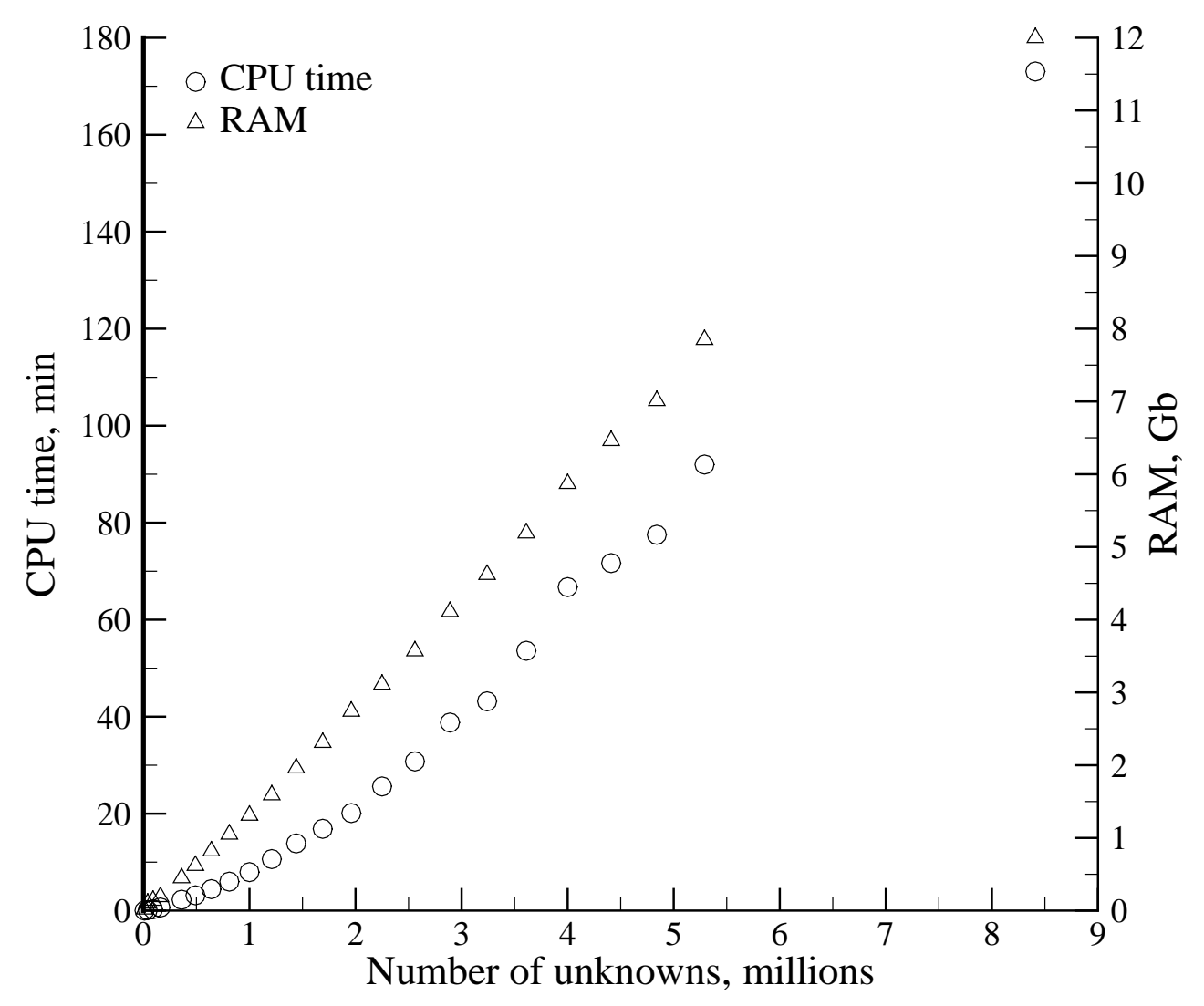

Fig. 3. Solver (VSS) statistics for a rigid-wall duct.

physically more meaningful quantity than the error norm of the computed solution because it is the quantity that is perceived by the human ear as the noise source propagates down the duct. This metric has units of decibels, and is defined as

$$
\begin{gathered}
\Delta \mathrm{dB}=10 \log _{10} \frac{E(0)}{E(L)} \\
E(x)=\int_{0}^{H} \frac{1}{2} \operatorname{Re}\left\{p(x, y) u^{*}(x, y)\right\} d y
\end{gathered}
$$

where the superscript asterisk denotes the complex conjugate, $\operatorname{Re}\{\}$ denotes the real part of the bracketed quantity, and $u$ is the normal component of acoustic particle velocity at axial location $x$. For the zero-flow calculations considered here, the acoustic velocity is related to 
the pressure gradient via the axial momentum equation

$$
u(x, y)=\frac{-1}{\left(2 i \rho_{0} \pi f c_{0}\right)} \frac{\partial p}{\partial x}
$$

It can be shown from the exact solution that no sound is attenuated in a rigid-wall duct (i.e., $\Delta \mathrm{dB}=0)$. The solution vector $\{\Phi\}$ obtained from the equation solver is used to compute the noise level, $\Delta \mathrm{dB}$, numerically. This metric is then used to access the accuracy of the solver solutions. These statistics are plotted as a function of the number of complex unknowns in Figure 4. Note that noise level predictions computed from the equation solver

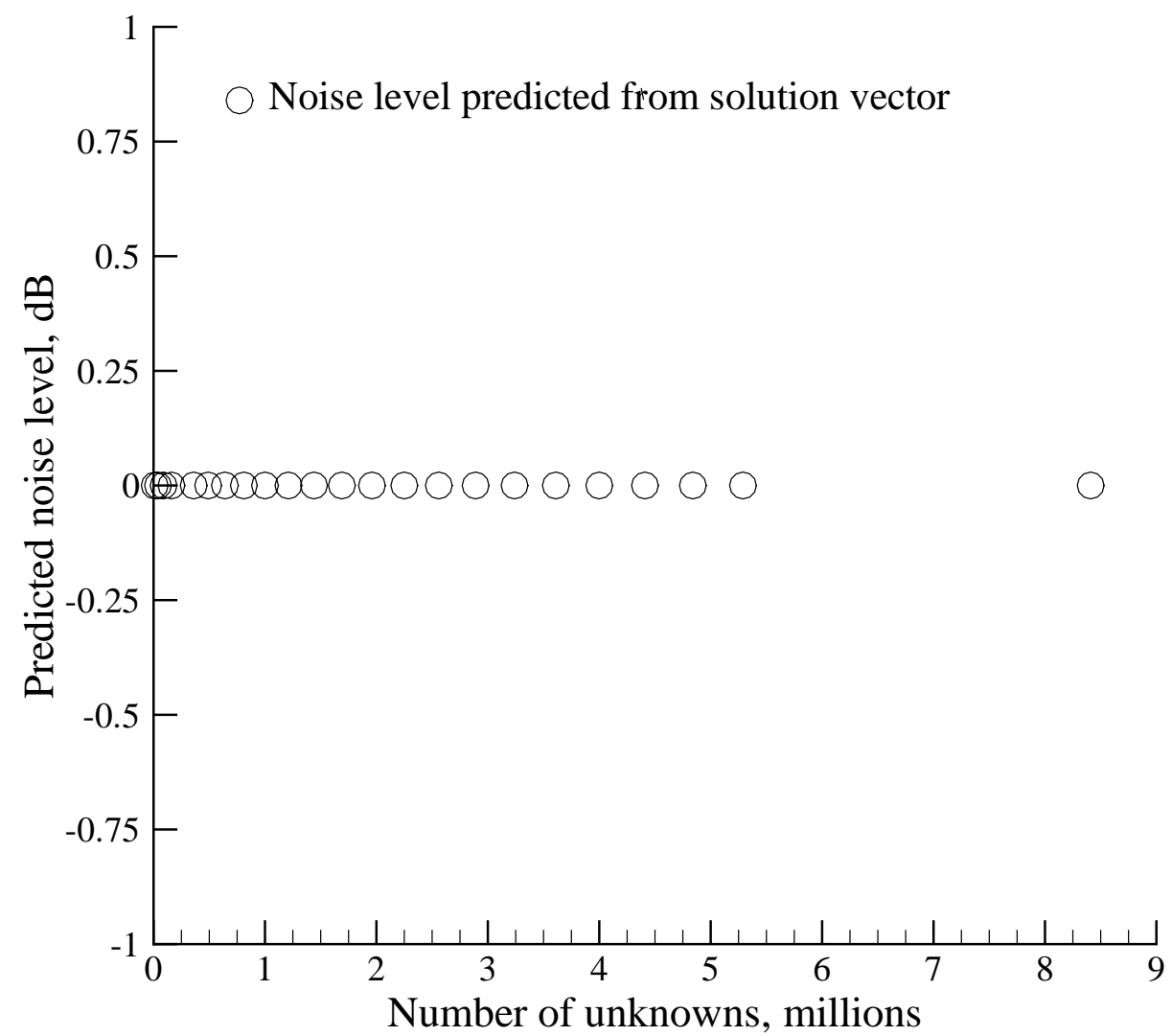

Fig. 4. Noise level predictions from the solver solution vector.

solution vector are in excellent agreement with the exact value of $0.0 \mathrm{~dB}$.

Statistics have also been computed for a soft-wall duct with identical dimensions as that of the rigid-wall duct, but with a wall impedance of $\zeta(x)=0.5-0.5 i$. The CPU time and 
RAM requirements were minimally affected by the presence of the liner. The statistics for the soft-wall duct are therefore not presented, since they were nearly identical to those given in Figure 2. All computations presented here were computed by setting the loop level parameter [7] to unity. This value of the loop level parameter was observed to be optimal for these zero-flow computations. It has been observed that the choice of the loop level parameter could have a significant impact on the performance of the solver. For example, when the statistics given in Figures 2 and 3 were computed with a loop level of 6 (i.e., the default loop level for the solver), both the CPU time and RAM requirements increased by a factor of 2 .

\section{Conclusions}

The commercial version of the NASA General-Purpose Solver has been exercised to solve several problems in nacelle aeroacoustics that did not contain flow. Statistics show that the solver is capable of solving 10 thousand complex unknowns in as little as 0.01 sec and 8.4 million complex unknowns in slightly less than 3 hours. The 8.4 million complex equation set represents an upper limit on the problem size that could be retained in memory (RAM) on an SGI ORIGIN 2000 with 12 Gb available RAM. The 8.4 million complex equation solution set also represents an upper limit of what would be expected in simulations of real laboratory experiments involving impedance eductions and is the largest aeroacoustics problem solved to date with this solution technique. This study was unable to detect any noticeable error in the solution, since noise levels predicted from the equation solver's solution vector (i.e., with as many as 8.4 million complex unknowns) are in excellent agreement with the decibel levels computed from the exact solution. Statistics for rigid-wall ducts show that the solver RAM requirements and CPU times scale with the first and $4 / 3$ power, respectively, of the number of unknowns. The performance of the solvers is minimally affected by the presence of the liner.

Results presented in [3] indicate that the matrix equation described by Equation (7) must 
be solved 20 to 40 times for successful impedance eductions at the low end of the impedance spectrum (i.e., frequencies below $3000 \mathrm{~Hz}$ ). Although the solver allows the computations to be extended to the high frequency end of the impedance spectrum where 8 million equations need to be solved, impedance eduction still appears to be impractical on a single processor because a single pass through the solver requires nearly 3 hours of central processing unit time. This research, therefore, supports a recommendation that efforts be made to exploit the multi-processor capability of the solver so that aeroacoustic optimization studies become practical for large-scale aeroacoustics computations. This recommendation is in concert with the NASA 256-processor SGI ORIGIN 2000 system (to be upgraded to 512 processors) of the same type used in this study.

\section{Acknowledgements}

The authors would like to gratefully acknowledge Dr. Majdi A. Baddourah of SolverSoft for his useful suggestions and assistance with VSS, the commercial version of the NASA GPS software.

\section{References}

[1] Bielak GW. Premo JW. Hersh AS. Advanced Turbofan Duct Liner Concepts. NASA CR-1999-209002, February, 1999.

[2] Watson WR. Tanner SE. Parrott TL. Optimization method for educing variableimpedance liner properties. AIAA Journal 1998; 36(1): 18-23.

[3] Watson WR. Jones MG. Parrott TL. Validation of an impedance eduction method in flow. AIAA Journal 1999; 37(7): 85-94.

[4] Varga RS. Matrix iterative methods. Englewood Cliffs, CA: Prentice-Hall, 1962.

[5] Fox L. An introduction to numerical linear algebra. New York: Oxford University Press, 1965. 
[6] Stead DJ. A best choice numerical method for large-scale computations in nacelle acoustics. MS Thesis, JIAF. Hampton VA: The George Washington University, 1999.

[7] Baddourah M. Solution of matrix equations using sparse technique, in The role of computers in research and development at Langley Research Center. NASA CP-10159, 1994: $130-146$.

[8] Storaasli OO. Performance of NASA equation solvers on computational mechanics applications, Proceedings of the 37th AIAA/ASME/ASCE/ANS/ASC structures, structural dynamics and materials conference 1996: 1680-1685.

[9] Kraft RE. Theory and measurements of acoustic wave propagation in multi-segmented rectangular ducts. Ph.D. Thesis, University of Cincinnati, 1976.

[10] Myers MK. On the acoustic boundary condition in the presence of flow. J. Sound and Vibr 1980; 71(3): 429-434. 